\title{
How genetic testing can lead to targeted management of XIAP deficiency-related inflammatory bowel disease
}

\author{
Ole Haagen Nielsen, DMSc ${ }^{1}$ and Eric Charles LaCasse, $\mathrm{PhD}^{2}$
}

X-linked lymphoproliferative disease type 2 (XLP-2, OMIM 300635) is a primary immunodeficiency caused by the loss of X chromosomelinked inhibitor of apoptosis (XIAP), the X-linked inhibitor of apoptosis gene at Xq25. XLP-2 individuals are susceptible to several specific and potentially fatal infections, such as Epstein-Barr virus (EBV). Children with XIAP-related XLP-2 may present with either familial hemophagocytic lymphohistiocytosis, often triggered in response to EBV infection, or with a treatment-refractory severe pediatric form of inflammatory bowel disease (IBD) that might be diagnosed as Crohn disease. However, this monogenic cause of IBD is distinct from adult Crohn disease (a polygenic and multifactorial disease) in its etiology and responsiveness to therapy. XLP-2 and the associated IBD symptoms are managed by a reduced-intensity conditioning regimen with an allogeneic hematopoietic stem cell transplantation that causes reso- lution of gastrointestinal symptoms. Exome sequencing has enabled identification of XIAP-deficient diseased individuals and has altered their morbidity by providing potentially lifesaving strategies in a timely and effective manner. Here, we summarize XLP-2 IBD treatment history and patient morbidity/mortality since its original identification in 2006. Since XLP-2 is rare, cases are probably undergiagnosed or misdiagnosed. Consideration of XLP-2 in children with severe symptoms of IBD can prevent serious morbidities and mortality, avoid unnecessary procedures, and expedite specific targeted therapy.

Genet Med advance online publication 14 July 2016

Key Words: apoptosis; Crohn disease; genetic counseling; immunodeficiencies; XIAP deficiency
X-linked lymphoproliferative (XLP) syndromes have only recently been recognized as recessive inherited primary immunodeficiencies, which are almost exclusively observed in males. ${ }^{1}$ XLP is divided into two distinct groups: XLP-1 (OMIM 308240), which is caused by mutations/loss of the signaling lymphocytic activation molecule-associated protein (SAP) in the gene SH2D1A encoding for the SAP protein, ${ }^{2}$ and XLP-2 (OMIM 300635), which is caused by $\mathrm{X}$ chromosome-linked inhibitor of apoptosis (XIAP) deficiency due to by mutations in the XIAP gene (previously referred to as the baculoviral IAP repeat containing 4 or BIRC4 gene) at the chromosomal locus Xq25 (ref. 3) (Table 1). Both of these primary immunodeficiencies are frequently associated with a macrophage activationlike syndrome known as hemophagocytic lymphohistiocytosis (HLH), and intermittent splenomegaly associated with cytopenia and fever is preferentially observed in XLP-2 (ref. 1). Although female carriers of X-linked recessive disorders typically do not have symptoms, a few heterozygous female carriers of XIAP mutations have developed symptoms reminiscent of their male counterparts that demonstrate an abnormal skewed inactivation toward the mutated allele in women carrying one altered allele of the XIAP gene., ${ }^{4,5}$

XLP-2 is usually, albeit not exclusively, considered in the setting of fulminant viral infection with Epstein-Barr virus (EBV) ${ }^{1,6}$ EBV is a ubiquitous virus reaching more than $90 \%$ of the adult population worldwide, ${ }^{7}$ which in healthy immunocompetent individuals only causes infectious mononucleosis, at worst. Under usual circumstances, EBV, after the acute infection, remains dormant within the host and is primarily harbored in B cells. ${ }^{8}$ The virus is generally inactive (latent) and controlled by other lymphocytes (T cells) specifically targeting EBV-infected B cells. ${ }^{9}$ However, individuals with XLP-2 may respond to EBV infections by producing abnormally large numbers of $\mathrm{T}$ cells, $\mathrm{B}$ cells, and macrophages that can cause primary HLH, a life-threatening immunodeficiency disorder with associated hyperinflammation. ${ }^{10}$ This results in a violent immune response with accumulation of these cells in organs, as well as an uncontrolled infection producing huge amounts of proinflammatory cytokines initiating tissue damage and organ failure, which can ultimately be fatal. ${ }^{6,11}$

XLP- 1 syndrome does not present with inflammatory bowel disease (IBD)-like intestinal symptoms (although affected individuals may develop lymphomas that often arise in the ileocecal region of the intestine) ${ }^{12}$ (Table 1). By contrast, symptoms involving the GI tract that mimic IBD and Crohn disease might in some cases be the exclusive disease manifestation of XIAP deficiency (i.e., XLP-2), and the diagnosis in those who have the mutation is reached solely with a confirmatory genetic test. ${ }^{5,13}$ Thus, the aim of this review is to emphasize to clinicians that XIAP deficiency should be considered in patients suspected to have Crohn disease with early onset or an aggravated clinical course resistant to conventional therapy, and that appropriate treatment strategies are available.

${ }^{1}$ Department of Gastroenterology, Medical Section, Herlev Hospital, University of Copenhagen, Herlev, Denmark; ${ }^{2}$ Apoptosis Research Centre, Children's Hospital of Eastern Ontario Research Institute, Ottawa, Ontario, Canada. Correspondence: Ole Haagen Nielsen (ohni@regionh.dk) 
Table 1 Clinical spectrum of presenting manifestations demonstrating phenotypic similarities and differences in patients with SAP deficiency (XLP-1) or XIAP deficiency (XLP-2)

\begin{tabular}{|c|c|c|c|}
\hline & XLP-1 & XLP-2 & Ref. \\
\hline \multicolumn{4}{|l|}{ Immunodeficiency and infection } \\
\hline $\begin{array}{l}\mathrm{HLH}^{\mathrm{a}} \text { (due to EBV, CMV, and other } \\
\text { infections) }\end{array}$ & ++ & ++ & $\begin{array}{c}3,29,36 \\
69,70\end{array}$ \\
\hline Splenomegaly & $+/-$ & ++ & 3,36 \\
\hline Hypo- or dysgammaglobulinemia & ++ & ++ & 12,70 \\
\hline $\begin{array}{l}\text { Severe or fulminant infectious } \\
\text { mononucleosis (due to EBV) }\end{array}$ & + & ++ & 12,53 \\
\hline Intestinal Crohn-like inflammation & - & ++ & $5,36,66,69$ \\
\hline Aplastic anemia & + & - & 71 \\
\hline $\begin{array}{l}\text { Other infectious or inflammatory } \\
\text { manifestations }^{b}\end{array}$ & + & + & 3 \\
\hline \multicolumn{4}{|l|}{ Biochemical or cellular defects } \\
\hline $\begin{array}{l}\text { Liver cytotoxicity/transaminitis and } \\
\text { dysfunction (related to hepatitis) }\end{array}$ & + & + & 3 \\
\hline $\begin{array}{l}\text { Increased and sustained serum IL-18 } \\
\text { (in association with HLH) }\end{array}$ & - & + & 72 \\
\hline $\begin{array}{l}\text { Increased or altered T-cell sensitivity to } \\
\text { AICD/RICDc }\end{array}$ & $+/-$ & + & 73 \\
\hline $\begin{array}{l}\text { Decreased NK/iNKT cell number or } \\
\text { function }\end{array}$ & + & $+1-$ & 36,53 \\
\hline $\begin{array}{l}\text { Defective fibroblast, monocyte, and } \\
\text { PBMC responses to NOD } 1 / 2 \text { ligands }\end{array}$ & - & + & 4,27 \\
\hline \multicolumn{4}{|l|}{ Lymphoproliferative disorders } \\
\hline $\begin{array}{l}\text { Malignant lymphoma (often seen in } \\
\text { the intestine } e^{d} \text { ) }\end{array}$ & ++ & - & 5,74 \\
\hline
\end{tabular}

Note that considerable phenotypic variability can be present even within a family carrying the same gene mutation.

+ , yes; ++, fairly typical; - , no; +/-, not typical or only partially affected or showing variable presentation at diagnosis.

AICD, activation-induced cell death; CMV, cytomegalovirus; EBV, Epstein-Barr virus; $\mathrm{HLH}$, hemophagocytic lymphohistiocytosis; IBD, inflammatory bowel disease; IL-18, interleukin-18; iNKT, invariant natural killer T cell; NK, natural killer cell; NOD1/2, nucleotide-binding oligomerization domain containing 1 or 2; PBMC, peripheral blood mononuclear cell; RICD, restimulation-induced cell death (i.e., another designation for AICD); XLP1, X-linked lymphoproliferative disease type 1 (OMIM 308240); XLP-2, X-linked lymphoproliferative disease type 2 (OMIM 300635).

aHLH diagnosis is established either by fulfilling a molecular genetic confirmation for a familial syndrome HLH gene mutation or by having five out of eight of the following criteria: (i) fever; (ii) splenomegaly; (iii) cytopenia (affecting more than two cell lineages); (iv) hypertriglyceridemia and/or hypofibrinogenemia; (v) hemophagocytosis in the bone marrow, spleen, or lymph nodes without evidence of malignancy; (vi) low or absent NK cell cytotoxicity; (vii) hyperferritinemia; and (viii) elevated soluble CD25/L2R. bSuch as prolonged or periodic pyrexia, hepatitis, skin abscesses, uveitis, or vasculitis. 'Opposite effects has been observed between SAP deficiency and XIAP deficiency. XLP1 T cells are more resistant than normal cells to AICD, whereas XLP-2 T cells are more sensitive to AICD than normal cells. ${ }^{d} S e e n$ primarily in the ileocecal region.

The table was compiled from the following references: ${ }^{3-5,12,27,29,36,53,66,69-74}$.

\section{SEARCH STRATEGY AND SELECTION CRITERIA}

The search "BIRC4, hemophagocytic lymphohistiocytosis, SAP protein, XIAP, X-linked IAP, XIAP deficiency OR XLP-2" in combination with "inflammatory bowel disease, Crohn disease OR ulcerative colitis" was performed in the PubMed and Embase databases (cutoff date: 3 May 2016) to determine the extent of XLP-2 with inflammation of the gastrointestinal tract. English-language reviews, practical guidelines, letters, editorials, and articles were evaluated. Subsequently, articles were selected according to their relevance as judged by the two authors, and additional papers were found in the articles' reference lists. For some of the clinical data presented in Table 2, it was necessary to contact the corresponding authors to clarify information.

\section{XIAP: IMMUNITY, NOD2 SIGNALING, AND IMMUNODEFICIENCY}

The BIRC4 gene encodes a caspase-inhibitory protein (XIAP) that protects cells from undergoing apoptosis in response to various death signals, including those coming from activated immune cells. ${ }^{14}$ In addition, XIAP mediates many aspects of innate immunity signaling and inflammation through XIAP's E3 ubiquitin ligase activity located in the C-terminal portion of the protein ${ }^{15}$ (Figure 1). XIAP's influence on receptor-interacting proteins, such as receptor-interacting protein 2 (RIP2), and inflammasome complexes allows either activation of the transcription factor NF- $\mathrm{KB}$ or generation of proinflammatory cytokines such as interleukin (IL)- 1 and IL-18. ${ }^{16}$ XIAP deficiency may also preclude lymphomagenesis, which could normally depend on antiapoptotic effectors like XIAP for cancer cell survival and disease progression. ${ }^{15,17}$

Importantly for IBD, XIAP is required for optimal signal transduction of the nucleotide-binding and oligomerization domain (NOD)-2 pathway, a pathway critical to intestinal immunity and susceptibility for Crohn disease. ${ }^{18,19}$ The binding of bacterial products, such as muramyl dipeptide (MDP), to NOD2 triggers self-association of the intracellular NOD2 receptor and the recruitment of a scaffolding protein, RIP2, and its polyubiquitination by XIAP and possibly E3 ubiquitin ligases, including Pellino3 or other IAPs such as cIAP1 and cIAP2 (refs. 19-23).The polyubiquitinated RIP2 acts as a signaling complex (or signalosome) by attracting another ubiquitin ligase, the linear ubiquitin assembly complex (LUBAC), and kinases, such as the IKB kinase kinase (IKK), leading to their proximityinduced phosphorylation and kinase activation. Both LUBACmediated Met1-linked ubiquitin chains and XIAP-mediated Lys63-linked ubiquitin chains are individually required for productive NOD2 signaling. ${ }^{24}$ This is highly reminiscent of the RIP1 signalosome that forms on tumor necrosis factor- $\alpha$ (TNF- $\alpha$ ) signaling through the TNF receptor that is dependent on RIP1 polyubiquitination by cIAP1 and cIAP2 (ref. 25). Activation of the kinases downstream of RIP2 (or RIP1) leads to the nuclear translocation of the transcription factor NF- $\kappa B$ and its gene modulatory effects on cytokine, chemokine, and defensin production to combat infections (Figure 2). XIAP gene mutations in XLP-2 individuals (Figure 3 ) are loss-of-function mutations that either lead to an absence of XIAP protein (decrease in the amount of full-length XIAP protein produced) or alter the function of critical regions (BIR2 and RING domains) in the expressed mutated protein that are required for XIAP-mediated NOD2 signaling in response to bacterial ligands (Figure 2). Hence, NOD2 signaling is severely compromised in XIAP-deficient individuals, leading 


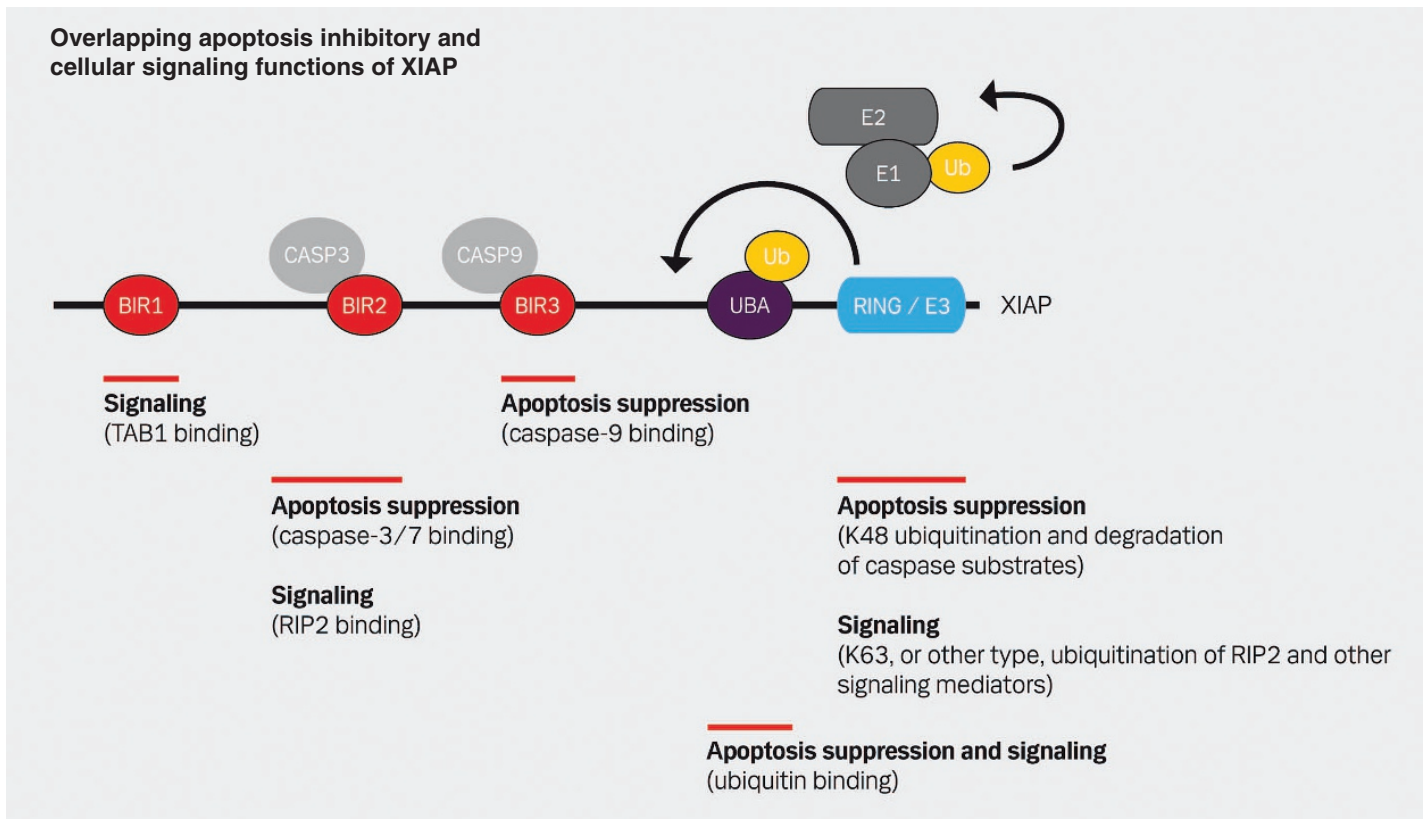

Figure 1 Influence of $X$ chromosome-linked inhibitor of apoptosis (XIAP) in overlapping apoptosis inhibitory functions. Many domains of the XIAP protein support overlapping roles in immune signaling and in apoptosis inhibition, some of which are illustrated here for the BIR (baculovirus IAP repeat), UBA (ubiquitin associated), and RING (really interesting new gene) protein domains. The RING domain is an E3 ubiquitin ligase that acts along with an E2 and E1 complex to tag XIAP substrates with K48-branched, K63-branched, or possibly other branched ubiquitin chains. This overlap of functions may explain why mutations leading to XIAP loss of function or deficiency can have different effects on immunity and disease presentation. For example, mutations can result in loss of apoptosis suppression and therefore sensitize NK and T cells to activation-induced cell death and compromise immunity. Alternatively, mutations can impair NOD2 signaling and downstream inflammatory responses. NK- and T-cell responses may be more important to antiviral defenses (such as against EBV), whereas NOD2 signaling is more critical for antibacterial defenses in the gut and in the prevention of inflammatory bowel disease. The loss of XIAP protein function or expression is probably compensated for by other RIP2-binding E3 ubiquilin ligases, such as CIAP1, cIAP2, and Pellino3, but only partially compensated for because disease still manifests with XIAP deficiency.

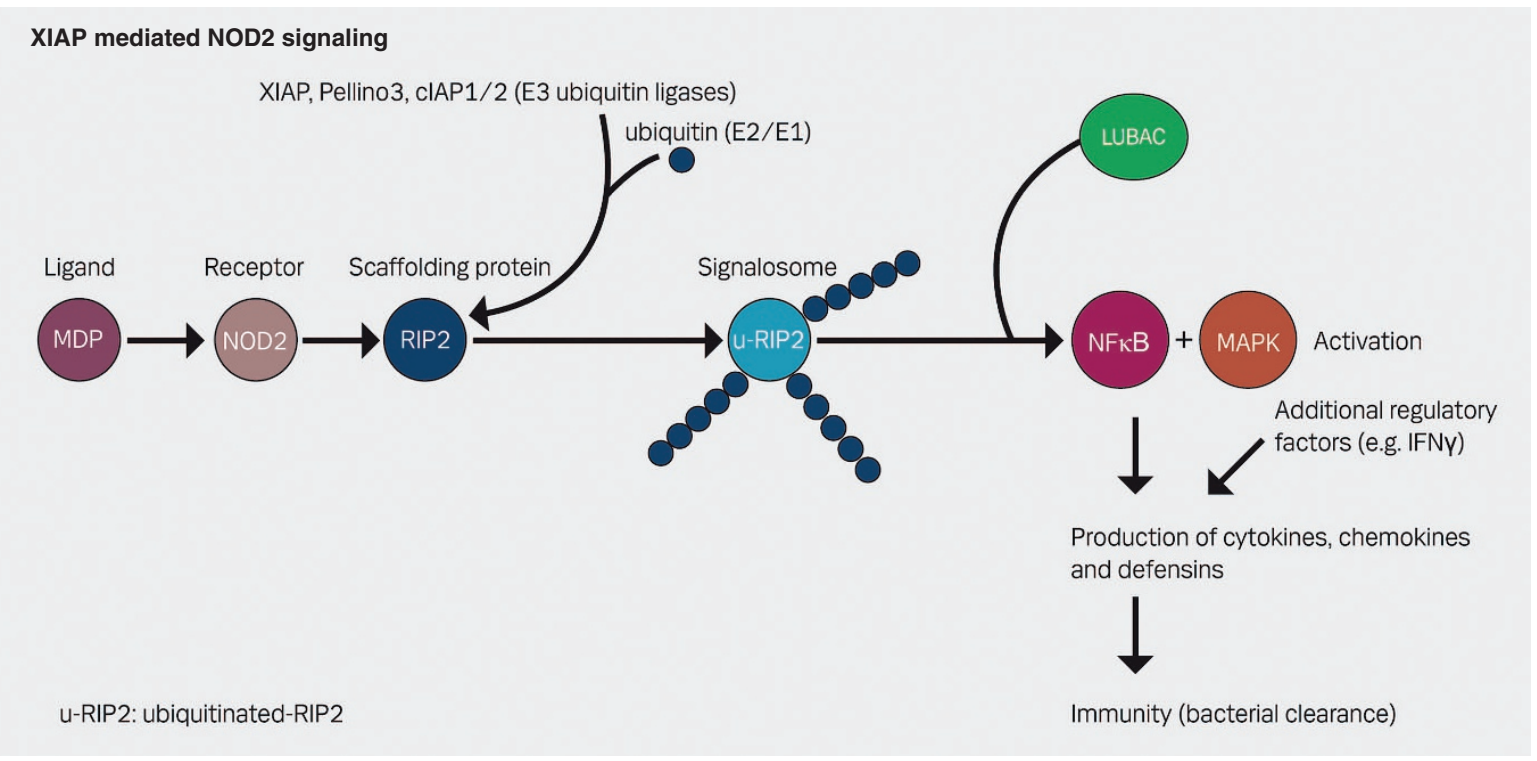

Figure 2 Role of $X$ chromosome-linked inhibitor of apoptosis (XIAP) in mediating NOD2 innate immune signaling. The recruitment of XIAP (and possibly other ubiquitin E3 ligases as well) to RIP2, upon NOD2 receptor stimulation by its ligand muramyl dipeptide (MDP), is shown. The XIAP-mediated Lys63 polyubiquitination of RIP2 (U-RIP2: ubiquitinated RIP2) creates a "signalosome," which allows the recruitment of the ubiquitin ligase complex LUBAC, which in turn leads to additional Met1, or linear, ubiquitination of the attached chains. This dual ubiquitination of RIP2 leads to recruitment and activation of kinases and the subsequent activation of NF-kB and MAPK signaling. The translocation of nuclear factor-kB factors to the nucleus leads to the transcriptional induction of genes encoding cytokines, chemokines, and defensins. Then innate inflammatory response also recruits neutrophils and other immune cells to the site to help control the infection. 


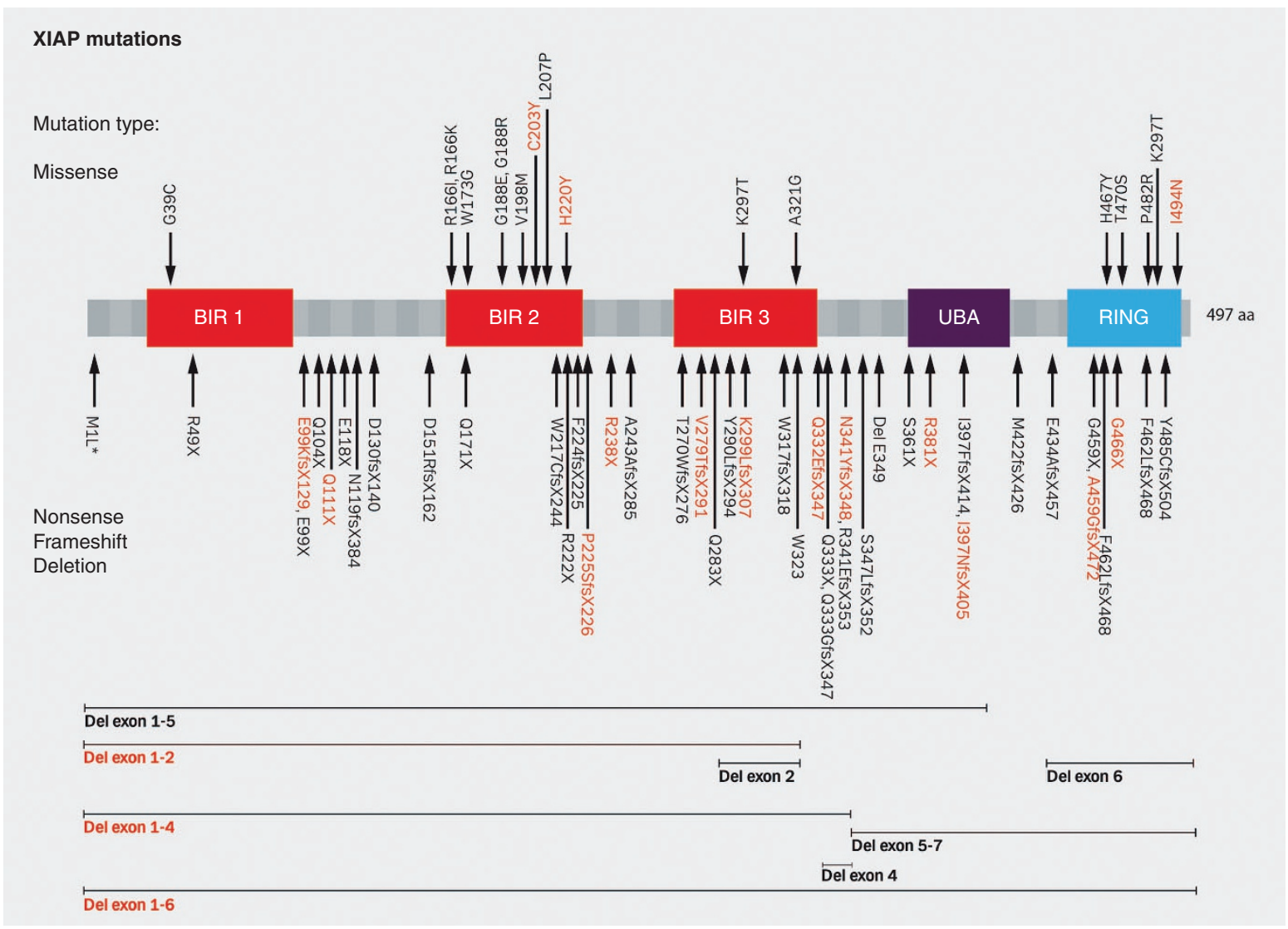

Figure 3 Overview of various deleterious mutations reported in the XIAP (BIRC4) gene in patients with XLP-2 (or in X chromosome-linked inhibitor of apoptosis (XIAP) deficiency presenting solely with inflammatory bowel disease (IBD)). Positions of the various XIAP mutations are shown against the diagram of the 497-amino-acid polypeptide-encoding human XIAP. Also shown are the various structural and functional homology domains in XIAP. These illustrated domains include the three BIR motifs, a zinc-finger domain that mediates many of the XIAP binding partner interactions, including binding and inhibition of caspases. The RING domain is a zinc-finger domain with E3 ubiquitin ligase activity, and the intervening UBA domain also participates in the recognition of ubiquitin and in the ubiquitination process. Missense mutations preferentially target the BIR2 region needed for binding to RIP2 and the RING domain needed for ubiquitination of RIP2 to mediate NOD2 signaling. The majority of the identified mutations may result in no XIAP protein being made or in a truncated polypeptide lacking the critical C-terminal RING domain. The 14 XIAP mutations identified in IBD patients in Table 2 are also shown here in red, as well as some additional reports of single case studies described in the text. *The missense mutation affecting the starting codon methionyl residue is included with the nonsense, frameshift, and deletion mutations because of the likely consequence of altering that critical initiating codon.

to a high degree of susceptibility to IBD developing with early onset and with a high degree of gene penetrance. ${ }^{26-28}$ Further, XIAP has recently been identified to play a role in fungal immunity through the dectin-1 pathway, because XIAP-deficient mice exhibit an XLP-2-like inflammatory syndrome on systemic Candida infection. ${ }^{28}$ However, it remains to be clarified whether this pathway also contributes to human disease in the case of XIAP deficiency.

The mechanism by which the lack of a functional XIAP protein results in the constellation of signs and symptoms of XLP-2 is not completely clear. However, evidence points to several likely possibilities to account for these manifestations, for example, the increased susceptibility of immune natural killer (NK) and innate mucosal-associated invariant T (MAIT) cells to apoptose (which may lead to EBV-associated HLH and splenomegaly), as well as defective monocyte/macrophage NOD2 signaling, which fails to control gut microbial homeostasis leading to manifestations of IBD. Nevertheless, in association with a serum cytokine storm - with or without EBV—or with other viruses, such as cytomegalovirus (CMV) or human herpes virus 6 (HHV6) exposure, ${ }^{29}$ macrophages might become inappropriately activated and may phagocytize bone marrowderived immune or erythroid cells, observed histologically as HLH. The proliferation of immune cells can, however, in the context of XLP-2, be classified as an X-linked familial HLH syndrome, with similarities to other familial HLH syndromes with defects in NK cell functions affecting granzyme B and perforin degranulation and with the reduction of NK cytotoxicity toward infected target cells. ${ }^{29,30}$ This complication, which is characterized by an uncontrolled immune activation of macrophages with an overproduction of cytokines such as IL-6 and IFN- $\gamma^{10}{ }^{10}$ produces clinical symptoms in association with recurrent fever, splenomegaly, or even hepatitis., ${ }^{3,10} \mathrm{HLH}$ is found in various forms of severity, ${ }^{6}$ for example, splenomegaly alone without any systemic signs (i.e., partial HLH), ${ }^{31}$ although in some cases it might take a fulminant course and be potentially lethal. ${ }^{6}$ 


\section{EPIDEMIOLOGY}

No epidemiological data regarding XLP syndromes are available, but XLP- 2 cases constitute approximately $40 \%$ of wellestablished XLP syndrome patients, ${ }^{1}$ without any racial or ethnic predilection..$^{32}$ Nevertheless, XLP-2 may be overlooked or even misclassified ${ }^{13}$ because current assessments suggest that $4 \%$ of early-onset IBD cases considered Crohn disease are, in fact, XIAP deficiency. ${ }^{33,34}$

Although clinical experience with patients known to have XLP-2 syndrome (first described in 2006 (ref. 35)) is limited, patients with XLP-2 syndrome have a slightly advantageous prognosis compared with XLP- 1 . Of the approximately 100 XIAP-deficient patients identified in a recent report, 22\% have died prematurely. ${ }^{36}$ Thus, individuals with HLH complications may experience a shortened life expectancy, ${ }^{3}$ although no increased rates of malignant lymphoma are observed in XLP-2 (ref. 5).

Table 2 summarizes the published clinical characteristics with comprehensive clinical data for 23 male individuals with Crohn disease-like symptoms. This cohort encompasses most of the XIAP-deficient IBD patients described in the literature (with a few exceptions). These patients have a $26 \%$ mortality rate, dying within a few years of onset of IBD symptoms or diagnosis. Of the six deaths, four were due to IBD, one to $\mathrm{HLH}$, and one to medical treatment (i.e., complications due to hematopoietic stem cell transplantation (HSCT)). The severity of IBD in XIAP-deficient individuals is highlighted by the facts that nearly half (i.e., 45\%) of the individuals have undergone abdominal surgery due to their bowel inflammation (Table 2) and that the median age of onset of IBD symptoms is 7 years (range, 0.1-41 years). By contrast, true IBD, or Crohn disease, as compared with symptoms related to XIAP-deficiency, is typically diagnosed later in life-after the age of $20 .{ }^{37}$ Thus, Crohn disease has been shown to lead to abdominal surgery in 38 and $60 \%$ at 5 and 30 years after diagnosis, respectively, although most data are from the prebiologic treatment era; ${ }^{38}$ however, because more efficient biologic therapies have been introduced in later years, this rate might decline in the future.

Furthermore, HLH seldom occurs in IBD, ${ }^{39}$ whereas isolated splenomegaly is unrelated to Crohn disease. Table 2 indicates that disease presentation is quite variable, even for members of the same family carrying the same XIAP mutation. A recent study in a Japanese cohort of 17 children with XLP-2 reported that six (35\%) of the individuals suffered from colitis, and one patient died due to bowel disease at the age of $4 .^{40}$ Another patient with colitis underwent abdominal surgery (a colostomy) and one received HSCT. All XIAP mutations are included in Figure 3.

\section{DIAGNOSIS OF XLP-2}

The rarity of Crohn disease during the first years of life and/ or resistance to conventional therapy should prompt a wider screening of such patients for possible other illnesses affecting immunologic or genetic defects. More than 50 distinct deleterious XIAP mutations have been found along all coding exons (cf. Figure 3), including missense and nonsense mutations as well as deletions and insertions. ${ }^{27}$ These comprise 29 mutations of the XIAP gene reported in a Japanese study as well. ${ }^{41}$

For identification of gene mutations, "next-generation" wholeexome DNA sequencing or sequencing of specific candidate disease genes might be used to sequence XIAP and other IBD candidate genes. These methodologies make it possible to capture all the possible XIAP mutations, including those not yet reported in the literature. Moreover, next-generation (DNA) sequencing techniques enable multiplexed sequencing of additional candidate disease genes at very little additional cost, such as can be done with existing small or large gene panels for IBD or for primary immunodeficiencies or genetic disorders in general. For example, a recent report describes the use of a 236-gene panel for primary immunodeficiencies (including several genes for monogenic IBD disorders) that identified a novel XIAP-deficiency mutation (Q111X) and that supports the benefits of using whole-exome sequencing to distinguish IBD secondary to a primary immunodeficiency ${ }^{42}$ In another study, 21 patients presenting with macrophage activation syndrome (MAS) were screened for primary HLH, and 7 of them were sent for genetic testing of candidate genes; 1 was identified as having XIAP deficiency (S361X). ${ }^{43}$

Genetic variants (polymorphisms and mutations) identified by sequencing can also be confirmed to be disease-causing by immunoblotting patients' peripheral white blood cells for XIAP protein levels and protein size. Alternatively, flow cytometric screening of permeabilized peripheral blood mononuclear cells for intracellular XIAP immunoreactivity might be applied. ${ }^{44}$ This method offers a quick XLP-2 screen with appropriate sensitivity and specificity. ${ }^{45}$ Most loss-of-function mutations of the birc4 gene appear to result in the loss or truncation of XIAP protein. However, the following caveat exists in that immunoblotting or flow cytometry should not be considered a conclusive diagnostic test for XLP-2 because point mutations, which might disrupt critical XIAP functions, may still lead to the appearance of full-length protein on a western blot or may preserve the epitope identified by flow cytometry, even though the XIAP protein is nonfunctional. Therefore, cellular functional assays of NOD2 signaling can be used to ascertain the function of XIAP variants in patient monocyte cell cultures treated with NOD2 agonists to facilitate the diagnostic process as well. ${ }^{46}$ This assay is helpful not only for IBD cases but also for HLH, because it functionally demonstrates the loss of XIAP protein. Nevertheless, there is still the possibility that point mutations outside of the BIR2 and RING domains (important for NOD2 signaling) contribute to HLH via other immune pathways, but this remains to be clarified. Note that such assays are considered experimental.

\section{XLP-2 SIMILARITIES TO CROHN IBD}

IBD consists of various disorders, of which ulcerative colitis ${ }^{47}$ and Crohn disease ${ }^{48}$ are the two most prevalent entities. In recent years, genome-wide association studies have identified more than 160 IBD susceptibility loci involved in the regulation of intestinal barrier functions as well as innate and adaptive 


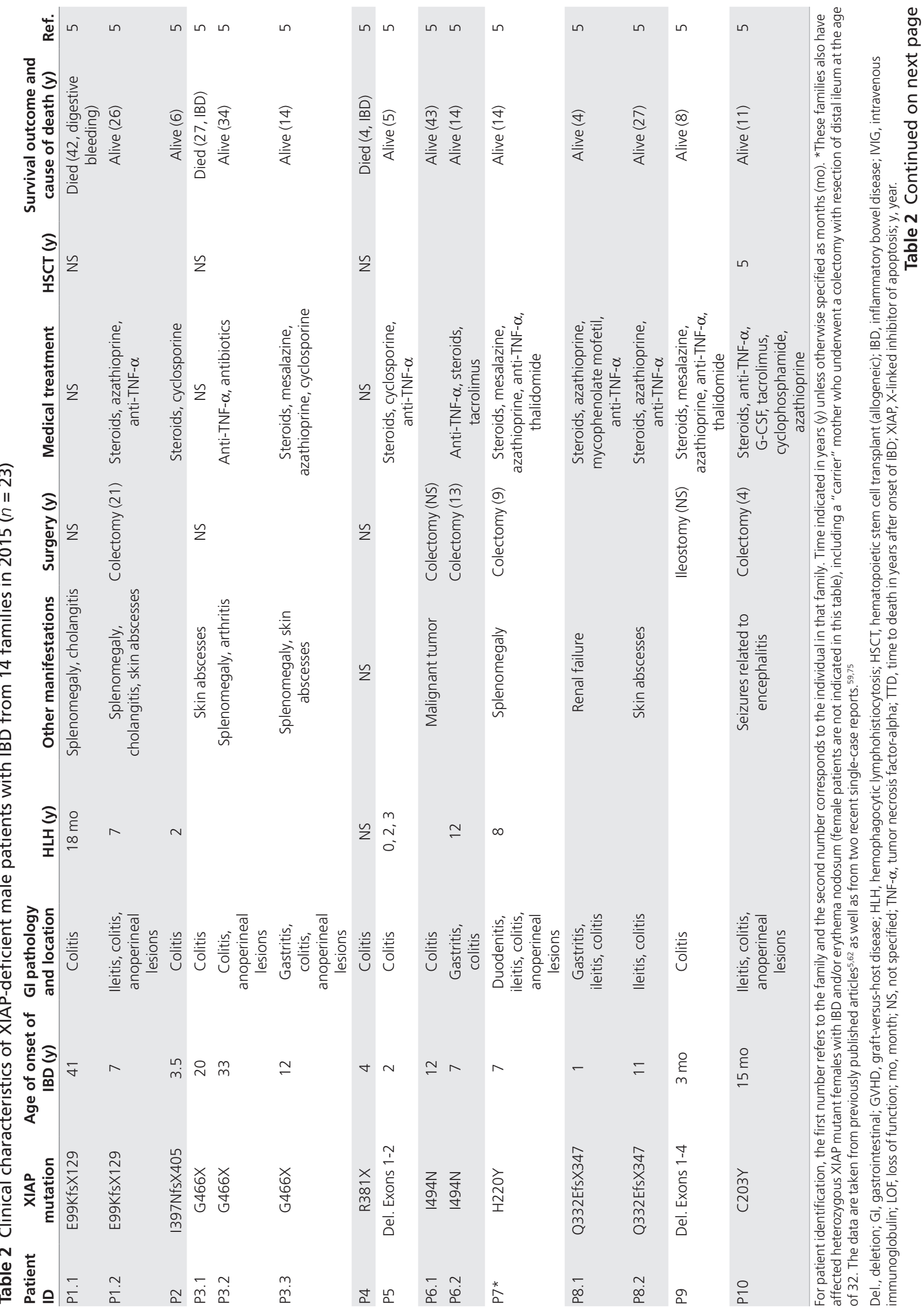




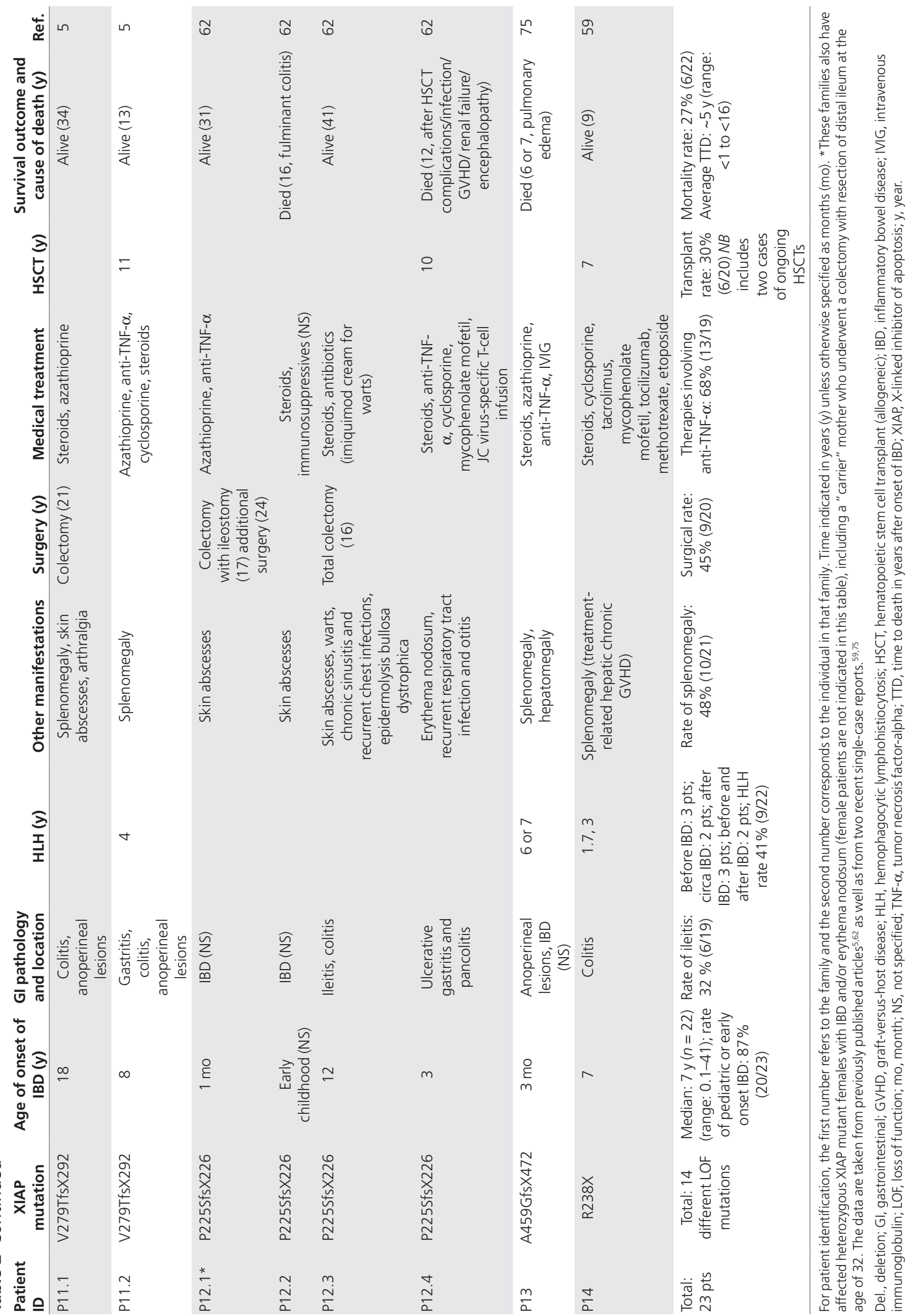


immunity, pointing to a close relationship between disturbance of the immune system and IBD. ${ }^{49}$ IBD is a clinical trait associated with several primary immune deficiencies comprising chronic granulomatous disease. In this context, it is interesting that immunomodulatory therapy with thiopurines (used for controlling IBD $)^{50}$ may trigger the development of $\mathrm{HLH},{ }^{39}$ and TNF inhibitor-induced skin lesions that might mimic manifestations observed in XIAP-deficient patients, ${ }^{36}$ such as eczema, pustolosis, psoriasis, and xerosis cutis together with infections (e.g., bacterial folliculitis, impetigo, and herpes zoster), are well-recognized complications. ${ }^{51}$

Striking similarities in the pathological mechanisms between XLP-2 and IBD have been observed that affect the intestinal epithelial barrier function. Thus, patients with XLP-2 have clinical manifestations and gut histology resembling Crohn disease, ${ }^{5,13}$ probably due to compromised NOD2 signaling (NOD2 receptors recognize peptidoglycan products such as MDP derived from bacterial cell walls) in intestinal immune cells. ${ }^{18,27}$ However, other possibilities for disease pathology exist owing to the loss of antiapoptotic activity of XIAP in the MAIT cells, for example, but further studies are needed to address these possibilities. Nevertheless, NOD2 is the strongest susceptibility gene associated with Crohn disease, and patients with NOD2 mutations experience a more aggravated clinical course. ${ }^{52}$ XIAP is critically required for NOD2 receptor signaling through its interaction with RIP2, which is bound to NOD2. XIAP causes Lys63-linked polyubiquitination of RIP2 and enables recruitment of LUBAC, which is another ubiquitin ligase that adds Met1-linked ubiquitin chains to RIP2, and both of these ubiquitination reactions are required for NF- $\mathrm{KB}$ signaling. This causes an increased production of cytokine, chemokine, and antimicrobial peptides required for gut immunity (Figure 2). Thus, the molecular importance of XIAP in the NOD2 pathway might explain why patients with XIAP gene mutations clinically resemble Crohn disease, especially because a substantial portion of patients with XIAP mutations report, or present with, inflammation from all segments of the GI tract, ${ }^{5}$ including skip lesions and inflammatory features together with crypt abscesses, epithelioid granulomas, transmural inflammation, and perianal fistulas. ${ }^{1,5,6,41,53}$ However, with the reservation for the low number of observations, it seems that ileocecal affection is more infrequent in XLP-2 (approximately 33\%; Table 2) versus more than $80 \%$ in Crohn disease. ${ }^{54,55}$

Moreover, extraintestinal manifestations of IBD are rather common, ${ }^{56}$ among these, arthralgia, uveitis, and erythema nodosum have been reported in XLP-2 as well. ${ }^{6,46,53}$ Uveitis has also been reported in an XIAP-deficient child with HLH, and the authors noted that uveitis is linked to XLP-2 but not to other familial HLH syndromes. ${ }^{57}$

\section{TREATMENT}

The clinical course in the subset of IBD patients diagnosed with XIAP deficiency is often severe, and there is no evidence that more aggressive treatment with conventional therapeutics (that is, glucocorticoids and immunomodulators such as thiopurines and methotrexate $)^{50}$ and TNF inhibitors ${ }^{58}$ is effective or beneficial to this group ${ }^{5,34,53}$ (Table 2). In this context, it should be emphasized that standardized medical treatment options for XLP-2 do not exist but need to be established through working groups and possibly in future randomized, controlled trials; however, a key issue is that it is difficult to accomplish this for a rare or even overlooked disorder.

A patient who is thought to have Crohn disease with an aggravated clinical course and resistance to conventional therapy should be tested for XLP-2. A prompt diagnosis can avoid unnecessary surgical and diagnostic procedures and therapies and might facilitate the initiation of lifesaving allogeneic HSCT that will cause resolution of Crohn disease-like symptoms. HSCT restores XIAP expression and function in immune cells derived from donor bone marrow precursors. This treatment option has been used with success in XLP-2 patients with intestinal symptoms $\mathrm{s}^{59}$ and may prevent the acute onset of HLH due to XIAP deficiency. ${ }^{34,53}$

In addition, patients with XLP-2 as well as HLH should be monitored for EBV infection status and be screened routinely for hypogammaglobulinemia, which is transient in this condition and might require intravenous immunoglobulin (IVIG) treatment. ${ }^{12}$ Allogeneic HSCT should be considered only in the setting of XLP-2-related IBD or HLH. Moreover, a recent clinical trial in adult patients with treatment-refractory Crohn disease (without a genetically identified primary immunodeficiency) demonstrated that autologous HSCT is not superior to conventional treatment. ${ }^{60}$

There is no established consensus or guideline regarding the criteria for deciding when to proceed to allogeneic HSCT for XLP-2. Nevertheless, it is recommended that allogeneic HSCT be considered as early as possible for most patients with XLP-2..$^{61-63}$ However, some additional issues need to be considered. Considerable initial morbidity and mortality may be associated with donor HSCT in patients with XIAP mutations (versus other primary immunodeficiencies), owing primarily to XIAP-deficient individuals experiencing increased toxicity to a busulfan-containing conditioning regimen. ${ }^{64}$ However, the risks related to the HSCT are not as serious when using non-busulfan conditioning regimens, ${ }^{36,59,61,63,65}$ indicating that conditioning regimens with reduced toxicity are promising alternatives and that therapy-other than HLH-should be limited to selected individuals with symptoms of severe Crohn disease refractory to conventional treatment. Thus, there is an urgent need for transplantation protocols associated with reduced toxicity, and current data indicate that combining a minimal intensity immunoablative antibody-based conditioning regimen with a high stem cell and T-cell dose graft makes it possible to secure curative engraftment with low toxicity for XIAP deficiency. ${ }^{61,65}$ The fact that allogeneic HSCT from an XIAP-proficient individual is a lifesaving option for XIAP-deficient individuals with IBD clearly suggests that the disease-causing defect due to XIAP loss is within the immune effector cell population and not within enterocytes and other cell types (because the gut 
cells are still XIAP-deficient in the XLP-2 individuals who have undergone transplantation).

\section{SUMMARY}

XIAP deficiency-related intestinal inflammation may be indistinguishable from Crohn disease, and the contribution of XIAP mutations to intestinal inflammation and its effects on responsiveness to therapy and any other associations with morbidity or mortality should be thoroughly assessed. This syndrome may be more prevalent than previously thought, especially for the subset of Crohn disease patients with an early age of onset $(<18$ years old) or a more aggravated clinical course refractory to conventional medical therapy, or both.

In addition, identification of individuals carrying XIAP mutations will enable notification of those families and their physicians (as well as genetic counselors) so that they may informed decisions. Asymptomatic individuals (family members identified as carrying the same disease-causing mutation) may develop a fatal disease (e.g., HLH) related to their underlying primary immunodeficiency, which could be mitigated in part via patient education, attentive screening (e.g., EBV testing), and prevention (e.g., IVIG administration), as well as aggressive therapy with antivirals and other supportive care and eventually with allogeneic HSCT. Carrier females with this X-linked disorder would be counseled about the mode and likelihood of transmission to their offspring who may be carriers as well or may be affected, depending on the child's sex.

Diagnosis and treatment of these patients will require collaboration among a multidisciplinary team of physicians that includes pediatricians, gastroenterologists, geneticists, rheumatologists, immunologists/infectious disease specialists, intensivists, emergentologists, and hematologists. Diagnosis and targeted therapy can dramatically change the outcome for individuals with XLP-2, as seen in several case reports. Such an outcome was described in the case of a 15-month-old infant with intractable IBD, ${ }^{66}$ as well as for others (see Table 2). A recent report described a protracted diagnostic odyssey and treatment history for a child with very-early-onset IBD (presenting at 3 weeks of age) who underwent abdominal surgeries at the ages of 7 and 10. This patient was eventually diagnosed with XIAP deficiency (complete gene deletion, exons 1-6) at the age of 17 and referred for HSCT. ${ }^{67}$ Another detailed case report of very-early-onset IBD in an infant with XIAP deficiency encompassed the presentation of symptoms at 1 month of age to numerous interventions over the course of more than a year to an ultimate diagnosis of XIAP deficiency and allogeneic HSCT at 15 months. ${ }^{68}$ An earlier genetic diagnosis could have promoted timely HSCT and thus avoided a colectomy.

\section{CONCLUSION}

Recently reported results pertaining to XLP-2 (XIAP-deficient) individuals with IBD symptoms have provided us with a greater understanding of this primary immunodeficiency that leads secondarily to Crohn disease. The experiences gained in treating these individuals with reduced-intensity HSCT might facilitate improved survival and quality of life. Physicians and surgeons alike should be cognizant of this X-linked primary immunodeficiency syndrome so as to properly diagnose and treat it. This is especially important for individuals with early onset and an aggravated clinical course demonstrating resistance to conventional therapy. Guidelines need to be established for when to use lifesaving allogeneic HSCT with reduced-intensity conditioning regimens for XIAP-deficient individuals who present with, for example, IBD, HLH, or MAS-like syndromes.

\section{DISCLOSURE}

The authors declare no conflict of interest.

\section{REFERENCES}

1. Veillette A, Pérez-Quintero LA, Latour S. X-linked lymphoproliferative syndromes and related autosomal recessive disorders. Curr Opin Allergy Clin Immunol 2013:13:614-622.

2. Purtilo DT, Cassel CK, Yang JP, Harper R. X-linked recessive progressive combined variable immunodeficiency (Duncan's disease). Lancet 1975;1:935-940.

3. Pachlopnik Schmid J, Canioni D, Moshous D, et al. Clinical similarities and differences of patients with X-linked lymphoproliferative syndrome type 1 (XLP-1/SAP deficiency) versus type 2 (XLP-2/XIAP deficiency). Blood 2011;117: 1522-1529.

4. Yang $X$, Hoshino A, Taga $T$, et al. A female patient with incomplete hemophagocytic lymphohistiocytosis caused by a heterozygous XIAP mutation associated with non-random X-chromosome inactivation skewed towards the wild-type XIAP allele. J Clin Immuno/ 2015;35:244-248.

5. Aguilar C, Lenoir C, Lambert N, et al. Characterization of Crohn disease in $X$-linked inhibitor of apoptosis-deficient male patients and female symptomatic carriers. J Allergy Clin Immunol 2014;134:1131-41.e9.

6. Latour S, Aguilar C. XIAP deficiency syndrome in humans. Semin Cell Dev Biol 2015;39:115-123.

7. Cohen Jl. Epstein-Barr virus infection. N Engl J Med 2000;343:481-492.

8. Chaganti S, Ma CS, Bell Al, et al. Epstein-Barr virus persistence in the absence of conventional memory B cells: IgM+lgD+CD27+B cells harbor the virus in X-linked lymphoproliferative disease patients. Blood 2008;112:672-679.

9. Merlo A, Turrini R, Dolcetti R, et al. The interplay between Epstein-Barr virus and the immune system: a rationale for adoptive cell therapy of EBV-related disorders. Haematologica 2010;95:1769-1777.

10. Faitelson Y, Grunebaum E. Hemophagocytic lymphohistiocytosis and primary immune deficiency disorders. Clin Immunol 2014;155:118-125.

11. Usmani GN, Woda BA, Newburger PE. Advances in understanding the pathogenesis of HLH. Br J Haematol 2013;161:609-622.

12. Filipovich A, Johnson J, Zhang K, Marsh R. Lymphoproliferative disease, X-linked. http://www ncbi nlm nih gov/books/NBK1406/. Accessed 6 March 2016.

13. Speckmann C, Ehl S. XIAP deficiency is a mendelian cause of late-onset IBD. Gut 2014;63:1031-1032

14. LaCasse EC, Baird S, Korneluk RG, MacKenzie AE. The inhibitors of apoptosis (IAPs) and their emerging role in cancer. Oncogene 1998;17:3247-3259.

15. Beug ST, Cheung HH, LaCasse EC, Korneluk RG. Modulation of immune signalling by inhibitors of apoptosis. Trends Immunol 2012;33:535-545.

16. Pedersen J, LaCasse EC, Seidelin JB, Coskun M, Nielsen $\mathrm{OH}$. Inhibitors of apoptosis (IAPs) regulate intestinal immunity and inflammatory bowel disease (IBD) inflammation. Trends Mol Med 2014;20:652-665.

17. LaCasse EC. Pulling the plug on a cancer cell by eliminating XIAP with AEG35156. Cancer Lett 2013;332:215-224.

18. Strober W, Asano N, Fuss I, Kitani A, Watanabe T. Cellular and molecular mechanisms underlying NOD2 risk-associated polymorphisms in Crohn disease. Immunol Rev 2014;260:249-260.

19. Damgaard RB, Nachbur U, Yabal M, et al. The ubiquitin ligase XIAP recruits LUBAC for NOD2 signaling in inflammation and innate immunity. Mol Cell 2012;46:746-758.

20. Fekete T, Koncz G, Szabo B, Gregus A, Rajnavölgyi E. Interferon gamma boosts the nucleotide oligomerization domain 2-mediated signaling pathway 
in human dendritic cells in an X-linked inhibitor of apoptosis protein and mammalian target of rapamycin-dependent manner. Cell Mol Immuno/ 2015; e-pub ahead of print 2 November 2015.

21. Bertrand MJ, Doiron K, Labbé K, Korneluk RG, Barker PA, Saleh M. Cellular inhibitors of apoptosis CIAP1 and CIAP2 are required for innate immunity signaling by the pattern recognition receptors NOD1 and NOD2. Immunity 2009;30:789-801.

22. Krieg A, Correa RG, Garrison JB, et al. XIAP mediates NOD signaling via interaction with RIP2. Proc Natl Acad Sci USA 2009;106:14524-14529.

23. Yang S, Wang B, Humphries F, et al. Pellino3 ubiquitinates RIP2 and mediates Nod2-induced signaling and protective effects in colitis. Nat Immunol 2013;14:927-936.

24. Hrdinka M, Fiil BK, Zucca M, et al. CYLD limits Lys63- and Met1-linked ubiquitin at receptor complexes to regulate innate immune signaling. Cell Rep 2016;14:2846-2858.

25. Mahoney DJ, Cheung HH, Mrad RL, et al. Both CIAP1 and CIAP2 regulate TNFalpha-mediated NF-kappaB activation. Proc Natl Acad Sci USA 2008;105:11778-11783.

26. Uhlig HH, Schwerd T. From genes to mechanisms: the expanding spectrum of monogenic disorders associated with inflammatory bowel disease. Inflamm Bowel Dis 2016;22:202-212.

27. Damgaard RB, Fiil BK, Speckmann $C$, et al. Disease-causing mutations in the XIAP BIR2 domain impair NOD2-dependent immune signalling. EMBO Mo/ Med 2013;5:1278-1295.

28. Hsieh WC, Chuang YT, Chiang IH, Hsu SC, Miaw SC, Lai MZ. Inability to resolve specific infection generates innate immunodeficiency syndrome in Xiap-/- mice. Blood 2014;124:2847-2857.

29. Marsh RA, Madden L, Kitchen BJ, et al. XIAP deficiency: a unique primary immunodeficiency best classified as X-linked familial hemophagocytic lymphohistiocytosis and not as X-linked lymphoproliferative disease. Blood 2010;116:1079-1082.

30. Chandrakasan S, Filipovich AH. Hemophagocytic lymphohistiocytosis: advances in pathophysiology, diagnosis, and treatment. J Pediatr 2013; 163:1253-1259.

31. Vieth S, Ammann S, Schwarz K, et al. Clinical phenotype and functional analysis of a rare XIAP/BIRC4 mutation. Klin Padiatr 2013;225:343-346.

32. Nichols KE, Ma CS, Cannons JL, Schwartzberg PL, Tangye SG. Molecular and cellular pathogenesis of X-linked lymphoproliferative disease. Immunol Rev 2005:203:180-199

33. Amininejad L, Charloteaux E, Theatre $E$ et al. A candidate gene study of rare monogenic disorders with IBD-like phenotype identified rare variants in XIAP gene in a cohort of early-onset IBD patients. Gastroenterology 2015;148(suppl 1):S81-S82.

34. Zeissig Y, Petersen BS, Milutinovic S, et al. XIAP variants in male Crohn disease. Gut 2015;64:66-76.

35. Rigaud S, Fondanèche MC, Lambert N, et al. XIAP deficiency in humans causes an X-linked lymphoproliferative syndrome. Nature 2006;444:110-114.

36. Aguilar $C$, Latour $\mathrm{S}$. X-linked inhibitor of apoptosis protein deficiency: more than an X-linked lymphoproliferative syndrome. J Clin Immunol 2015;35: $331-338$.

37. Johnston RD, Logan RF. What is the peak age for onset of IBD? Inflamm Bowel Dis 2008;14(suppl 2):S4-S5.

38. Peyrin-Biroulet L, Harmsen WS, Tremaine WJ, Zinsmeister AR, Sandborn WJ, Loftus EV Jr. Surgery in a population-based cohort of Crohn disease from Olmsted County, Minnesota (1970-2004). Am J Gastroentero/ 2012;107:16931701.

39. Subramaniam K, D'Rozario J, Pavli P. Lymphoma and other lymphoproliferative disorders in inflammatory bowel disease: a review. J Gastroenterol Hepatol 2013;28:24-30.

40. Nishida N, Yang X, Takasaki I, et al. Dysgammaglobulinemia Associated With Glu349del, a Hypomorphic XIAP Mutation. J Investig Allergol Clin Immunol 2015;25:205-213.

41. Yang X, Kanegane H, Nishida N, et al. Clinical and genetic characteristics of XIAP deficiency in Japan. J Clin Immunol 2012:32:411-420.

42. Oh SH, Baek J, Kim KM, et al. Is Whole Exome Sequencing Clinically Practical in the Management of Pediatric Crohn Disease? Gut Liver 2015;9:767-775.

43. Cruikshank $\mathrm{M}$, Anoop P, Nikolajeva $\mathrm{O}$ et al. Screening assays for primary haemophagocytic lymphohistiocytosis in children presenting with suspected macrophage activation syndrome. Pediatr Rheumatol Online J 2014;12(suppl 1):48.
44. Marsh RA, Bleesing JJ, Filipovich AH. Using flow cytometry to screen patients for $X$-linked lymphoproliferative disease due to SAP deficiency and XIAP deficiency. J Immunol Methods 2010:362:1-9.

45. Gifford CE, Weingartner E, Villanueva J, et al. Clinical flow cytometric screening of SAP and XIAP expression accurately identifies patients with SH2D1A and XIAP/BIRC4 mutations. Cytometry B Clin Cytom 2014;86:263-271.

46. Ammann $S$, Elling $R$, Gyrd-Hansen $M$, et al. A new functional assay for the diagnosis of X-linked inhibitor of apoptosis (XIAP) deficiency. Clin Exp Immunol 2014;176:394-400.

47. Danese S, Fiocchi C. Ulcerative colitis. N Engl J Med 2011;365:1713-1725.

48. Baumgart DC, Sandborn WJ. Crohn disease. Lancet 2012;380:1590-1605.

49. Jostins L, Ripke S, Weersma RK, et al.; International IBD Genetics Consortium (IIBDGC). Host-microbe interactions have shaped the genetic architecture of inflammatory bowel disease. Nature 2012;491:119-124.

50. Nielsen $\mathrm{OH}$, Bjerrum JT, Herfarth H, Rogler G. Recent advances using immunomodulators for inflammatory bowel disease. I Clin Pharmacol 2013;53:575-588.

51. Cleynen I, Van Moerkercke W, Billiet T, et al. Characteristics of skin lesions associated with anti-tumor necrosis factor therapy in patients with inflammatory bowel disease: a cohort study. Ann Intern Med 2016;164:10-22.

52. Philpott DJ, Sorbara MT, Robertson SJ, Croitoru K, Girardin SE. NOD proteins: regulators of inflammation in health and disease. Nat Rev Immunol 2014;14: 9-23.

53. Speckmann C, Lehmberg K, Albert MH, et al. X-linked inhibitor of apoptosis (XIAP) deficiency: the spectrum of presenting manifestations beyond hemophagocytic lymphohistiocytosis. Clin Immunol 2013;149:133-141.

54. Munkholm P. Crohn disease-occurrence, course and prognosis. An epidemiologic cohort-study. Dan Med Bull 1997:44:287-302.

55. Caprilli R. Why does Crohn disease usually occur in terminal ileum? J Crohns Colitis 2008;2:352-356.

56. Larsen S, Bendtzen K, Nielsen $\mathrm{OH}$. Extraintestinal manifestations of inflammatory bowel disease: epidemiology, diagnosis, and management. Ann Med 2010;42:97-114.

57. Basiaga ML, Weiss PF, Behrens EM. BIRC4 Mutation: An Important Rare Cause of Uveitis. J Clin Rheumatol 2015;21:444-447.

58. Nielsen $\mathrm{OH}$, Ainsworth MA. Tumor necrosis factor inhibitors for inflammatory bowel disease. N Eng/ J Med 2013;369:754-762.

59. Tsuma $Y$, Imamura $T$, Ichise $E$, et al. Successful treatment of idiopathic colitis related to XIAP deficiency with allo-HSCT using reduced-intensity conditioning. Pediatr Transplant 2015;19:E25-E28.

60. Hawkey CJ, Allez M, Clark MM, et al. Autologous hematopoetic stem cell transplantation for refractory Crohn disease: a randomized clinical trial. JAMA 2015:314:2524-2534.

61. Chellapandian D, Krueger J, Schechter $T$, et al. Successful allogeneic hematopoietic stem cell transplantation in XIAP deficiency using reducedintensity conditioning. Pediatr Blood Cancer 2016;63:355-357.

62. Dziadzio M, Ammann S, Canning C, et al. Symptomatic males and female carriers in a large Caucasian kindred with XIAP deficiency. J Clin Immunol 2015:35:439-444.

63. Varghese AS, Lee H, Bonney D, Hughes S, Wynn R. Complications of reduced intensity conditioning HSCT for XIAP deficiency (alloimmune cytopenias and $\mathrm{HLH}$ ) successfully managed with donor lymphocyte infusion. J Pediatr Hematol Oncol 2015;37:e198-e199.

64. Marsh RA, Rao K, Satwani P, et al. Allogeneic hematopoietic cell transplantation for XIAP deficiency: an international survey reveals poor outcomes. Blood 2013;121:877-883.

65. Worth AJ, Nikolajeva O, Chiesa R, Rao K, Veys P, Amrolia PJ. Successful stem cell transplant with antibody-based conditioning for XIAP deficiency with refractory hemophagocytic lymphohistiocytosis. Blood 2013;121:4966-4968.

66. Worthey EA, Mayer AN, Syverson GD, et al. Making a definitive diagnosis: successful clinical application of whole exome sequencing in a child with intractable inflammatory bowel disease. Genet Med 2011;13: 255-262.

67. Kelsen JR, Dawany N, Martinez A, et al. A de novo whole gene deletion of XIAP detected by exome sequencing analysis in very early onset inflammatory bowel disease: a case report. BMC Gastroenterol 2015;15:160.

68. Girardelli M, Arrigo S, Barabino A, et al. The diagnostic challenge of very early-onset enterocolitis in an infant with XIAP deficiency. BMC Pediatr 2015;15:208 
69. Uhlig HH, Schwerd T, Koletzko S, et al.; COLORS in IBD Study Group and NEOPICS. The diagnostic approach to monogenic very early onset inflammatory bowel disease. Gastroenterology 2014;147:990-1007.e3.

70. Yang X, Miyawaki T, Kanegane H. SAP and XIAP deficiency in hemophagocytic lymphohistiocytosis. Pediatr Int 2012;54:447-454.

71. Sandlund JT, Shurtleff SA, Onciu M, et al. Frequent mutations in SH2D1A (XLP) in males presenting with high-grade mature B-cell neoplasms. Pediatr Blood Cancer 2013;60:E85-E87.

72. Wada T, Kanegane H, Ohta K, et al. Sustained elevation of serum interleukin-18 and its association with hemophagocytic lymphohistiocytosis in XIAP deficiency. Cytokine 2014;65:74-78.
73. Filipovich AH, Zhang $K$, Snow AL, Marsh RA. X-linked lymphoproliferative syndromes: brothers or distant cousins? Blood 2010;116:33983408.

74. Rezaei N, Mahmoudi E, Aghamohammadi A, Das R, Nichols KE. X-linked lymphoproliferative syndrome: a genetic condition typified by the triad of infection, immunodeficiency and lymphoma. Br J Haematol 2011;152:1 3-30.

75. Beșer ÖF, Conde CD, Kutlu T, Çullu Çokuğraş F, Boztuğ K, Erkan T. Inflammatory bowel disease with lethal disease course caused by a nonsense mutation in BIRC4 encoding X-linked inhibitor of apoptosis protein (XIAP). J Pediatr Gastroenterol Nutr 2016;62:e41-e43. 